\title{
Temperature Independence of the Droop Effect in Blue LED's Grown on Silicon Substrates
}

\author{
P. Tanner, J. Han, S. Dimitrijev \\ Griffith University \\ Queensland Micro- and Nanotechnology Centre \\ Nathan, Queensland, Australia, 4111. \\ Email: P.Tanner@griffith.edu.au
}

\begin{abstract}
Efficiency droop remains a significant problem to be overcome if the performance of LEDs for solid state lighting is to be improved. As more cost effective substrates such as silicon wafers are used on which to grow the LED active layers, the overall output efficiency and efficiency droop at high drive currents need to be monitored and better understood. This paper investigates the droop effect at elevated temperatures for an LED grown on silicon, compared with an LED grown on the more standard sapphire substrate. While the overall output efficiency decreases with increasing temperature, the efficiency droop is found to be relatively independent of temperature.
\end{abstract}

\section{Introduction}

Good progress has been made in recent years to improve efficiency and reduce production costs of LEDs. The growth of $\mathrm{AlGaN} / \mathrm{GaN}$ layers on large diameter silicon wafers can enable the cost reduction that is needed for a widespread adoption of blue/white LEDs for solid-state lighting. One of the biggest issues in blue LEDs is the reduction in efficiency at high input currents known as the "droop effect". Many papers are published about the droop effect for LED layers grown on sapphire, suggesting different mechanisms such as Auger recombination [1-3], electron leakage and recombination outside the active layers [4], and recombination at crystal defects [5] as possible causes. While much less is known about this effect in the recently commercialized blue LEDs created in $\mathrm{AlGaN} / \mathrm{GaN}$ layers grown on $\mathrm{Si}$ wafers, some studies are beginning to show similar effects [6,7]. Also, there is no widely accepted data about the impact of increased operating temperature on this effect.

In this paper, we present measurements of the output efficiency and droop effect at controlled temperatures for commercial blue LEDs grown on silicon and compare with commercial blue LEDs on sapphire substrates.

\section{Devices and Experimental Proceedures}

Two types of LED are compared. The first was grown on a silicon substrate, after which silicon was removed and the LED chip flipped so that light emission is through n-GaN. For comparison, a commercial LED grown on sapphire was also measured. Measurements were performed on a probe station with a temperature controlled sample stage. An Agilent B1505A parameter analyzer was used for I-V measurements and current pulsing. Light was detected by an Ocean Optics spectrometer mounted directly above the LED.

The forward bias current-voltage characteristics of the two LEDs are shown in Fig. 1 as linear (a) and log (b) plots. It can be seen that the characteristics are very similar, with both diodes exhibiting low current leakage up to a turn-on voltage of approximately $2.6 \mathrm{~V}$.
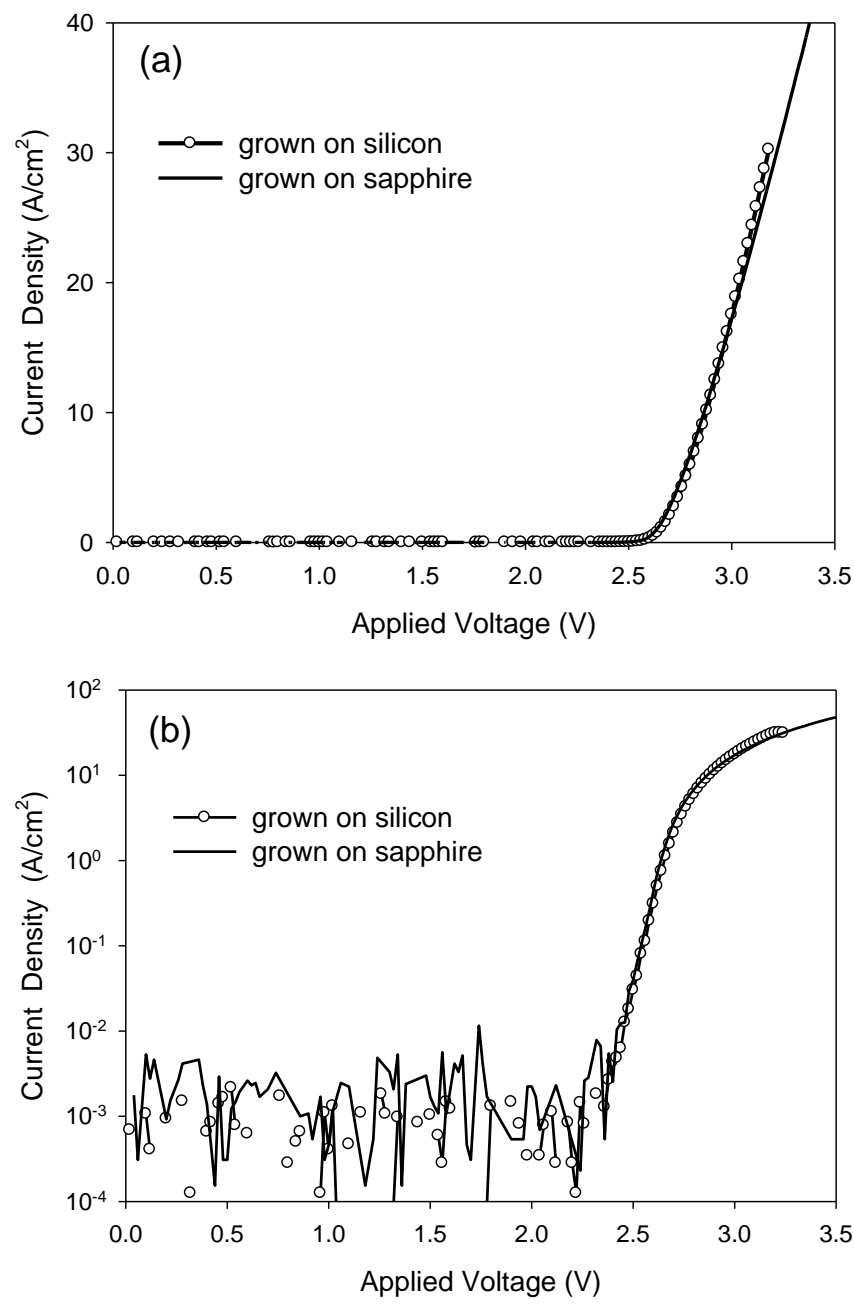

Fig. 1. Current-voltage curves of the two LED's measured at room temperature. (a) linear I-V and (b) Log I-V.

\section{Measurement Technique}

Devices were placed on a temperature controlled stage and connected to a measurement unit using four probes in the force/sense configuration to eliminate lead and probe resistance. With the spectrometer mounted directly above the device, the current was pulsed for $0.5 \mathrm{~s}$ with $4 \mathrm{~s}$ wait between pulses in order to reduce self-heating. The output spectrum at a low current density was observed at each temperature to determine the peak wavelength and then this wavelength was monitored as the current was pulsed. As shown in Fig 2, the 
response decays with time due to self heating and an observed red shift of the peak. Therefore, only the initial highest data point was used for each current pulse. At the same time, the applied voltage was monitored and thus the input power calculated and used to determine the output efficiency.

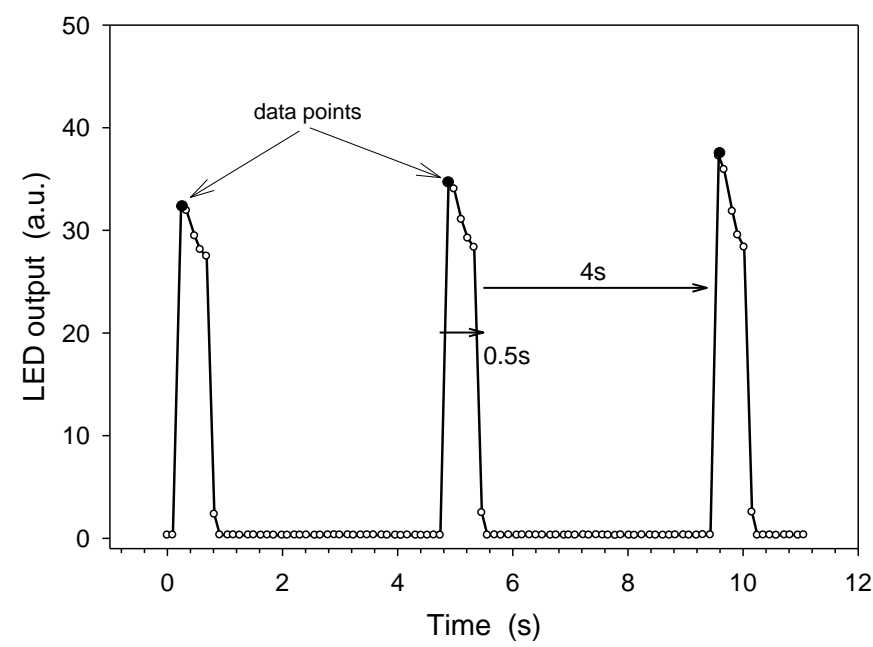

Fig. 2. Spectrometer output at the peak wavelength for a series of current pulses. Only the first data point (black dot) for each current pulse is used.

\section{Results and Discussion}

Fig. 3 compares the effect of increasing operating temperature and input current density on the light output efficiency of the two LEDs. Fig. 3(a) shows the results of the LED grown on silicon measured at different temperatures between $20^{\circ} \mathrm{C}$ and $150^{\circ} \mathrm{C}$. As the operating temperature increases to $150^{\circ} \mathrm{C}$, the maximum light output efficiency decreases by $57 \%$, and efficiency droop is clearly evident for higher input current densities. While this is clearly not desirable, Fig. 3(a) does indicate that the efficiency maximum shifts to higher current densities as the temperature increases. In comparison, the LED grown on sapphire, as seen in Fig. 3 (b), exhibits less decrease $(48 \%)$ in the maximum efficiency as well as less droop for the same temperature and current input range.

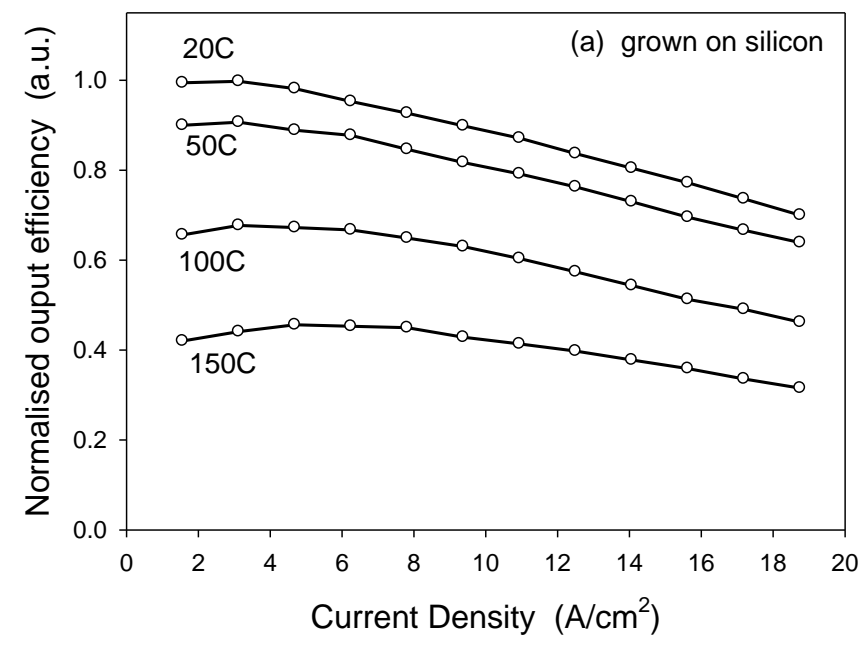

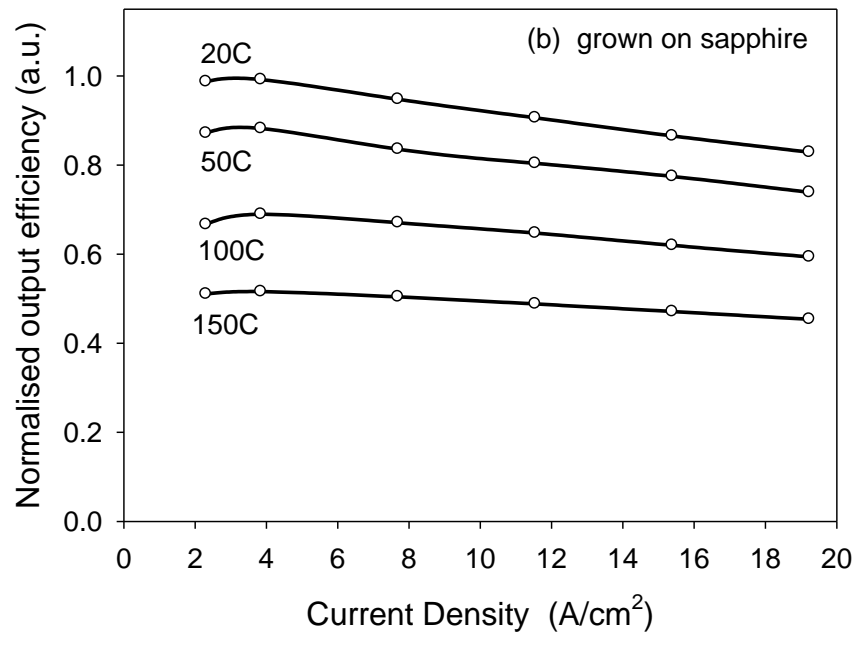

Fig. 3. Efficiency droop of LEDs grown on (a) silicon and (b) sapphire substrate measured at operating temperatures from $20^{\circ} \mathrm{C}$ to $150^{\circ} \mathrm{C}$.

When the percentage of efficiency droop is plotted as a function of temperature (Fig. 4) it can be seen that, as for the LED on sapphire, there is very little temperature dependence of droop for the LED grown on silicon. On the other hand, there is a significantly larger average droop of $31 \%$ for the LED grown on silicon, compared with $15 \%$ for the LED grown on sapphire. These differences in performance between the two LEDs may be related to the expected difference in crystal quality of the active layers.

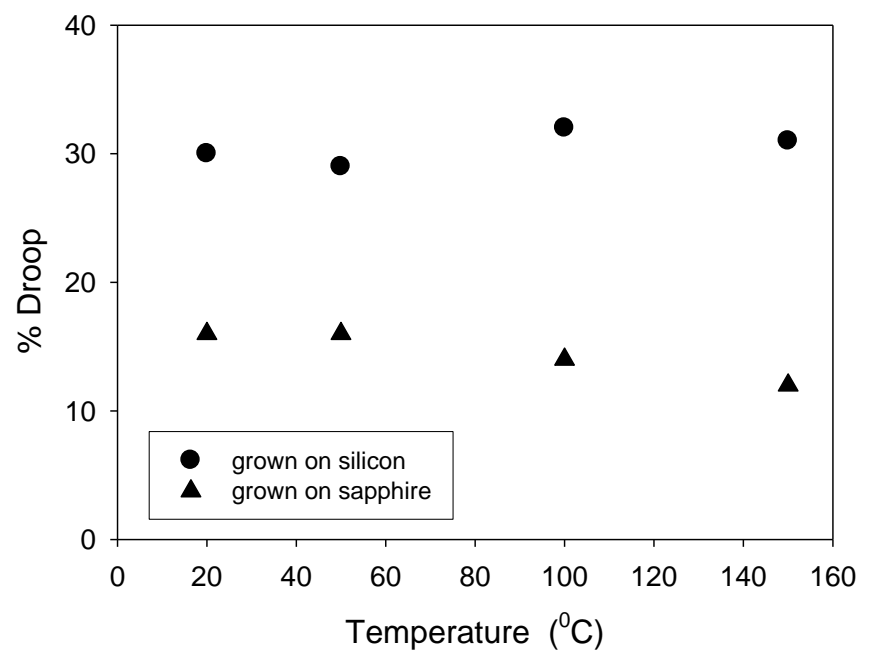

Fig. 4. The percent droop of output efficiency as a function of operating temperature for LEDs grown on silicon and sapphire.

\section{Summary}

This paper investigates the effect of operating temperature on the output efficiency and the efficiency droop of an LED grown on silicon and compares these parameters with a commercial LED with similar electrical characteristics grown on sapphire. Both devices exhibited very little temperature dependence of the efficiency droop. The maximum output efficiency of the LED grown on silicon decreased by $57 \%$ as 
its operating temperature was increased from $20^{\circ} \mathrm{C}$ to $150^{\circ} \mathrm{C}$, while the LED gown on sapphire performed slightly better with a $48 \%$ decrease. Measurements of the efficiency droop revealed a greater difference in performance, with values of $31 \%$ and $15 \%$ for the LEDs grown on silicon or sapphire respectively.

These results contribute to a better understanding of the efficiency droop effect of LEDs grown on silicon and may guide future investigations in this area. A number of techniques have been used to reduce the efficiency droop in LEDs grown on sapphire, including electron blocking layers, varying the width of the quantum wells, and the use of quaternary materials in the quantum wells. If these are also used for LEDs grown on silicon, similar improvements should result.

\section{References}

1. J. Iveland, L. Martinelli, J. Peretti, J. S. Speck, C. Weisbuch, "Direct Measurement of Auger Electrons Emitted from a Semiconductor Light-Emitting Diode under Electrical Injection: Identification of the Dominant Mechanism for Efficiency Droop", Phys. Rev. Let. 110, 177406, (2013).

2.Shen Y. C., Mueller G. O., Watanabe S., Gardner N. F., Munkholm A., Krames M. R., “Auger recombination in InGaN measured by photoluminescence", Appl. Phys. Letters., Volume 91, Issue 14, 141101 (2007).

3. M. Binder, A. Nirschl,R. Zeisel, T. Hager, H.-J. Lugauer, M. Sabathil, D. Bougeard, J. Wagner, and B. Galler, "Identification of nnp and npp Auger recombination as significant contributor to the efficiency droop in (GaIn)N quantum wells by visualization of hot carriers in photoluminescence", Appl. Phys. Letters., 103, 171108, (2013).

4. Kim M-H, Schubert M F, Dai Q, Kim J K, Schubert E F, "Origin of efficiency droop in GaN-based light-emitting diodes", App. Phys. Letters, 91, 183507 (2007).

5. Lin Y., Yong Zhang, Zhiqiang Liu, Liqin Su, Jihong Zhang, Tongbo Wei and Zhong Chen, "Spatially resolved study of quantum efficiency droop in InGaN light-emitting diodes", Appl. Phys. Letters, 101, 252103, (2012).

6. Liu M-L, Min Q-Y, Ye Z-Q, "Efficiency droop in blue InGaN/GaN light emitting diodes on Si substrate", Acta Phys. Sin. Vol. 61, No. 17, (2012).

7. Liu M-L, Ye Z-Q, Lei M-S, "Efficiency droop in blue InGaN/GaN single-quantum-well light-emitting diodes on the Si substrate", Semicond. Sci. Technology, 27, 045010, (2012). 